
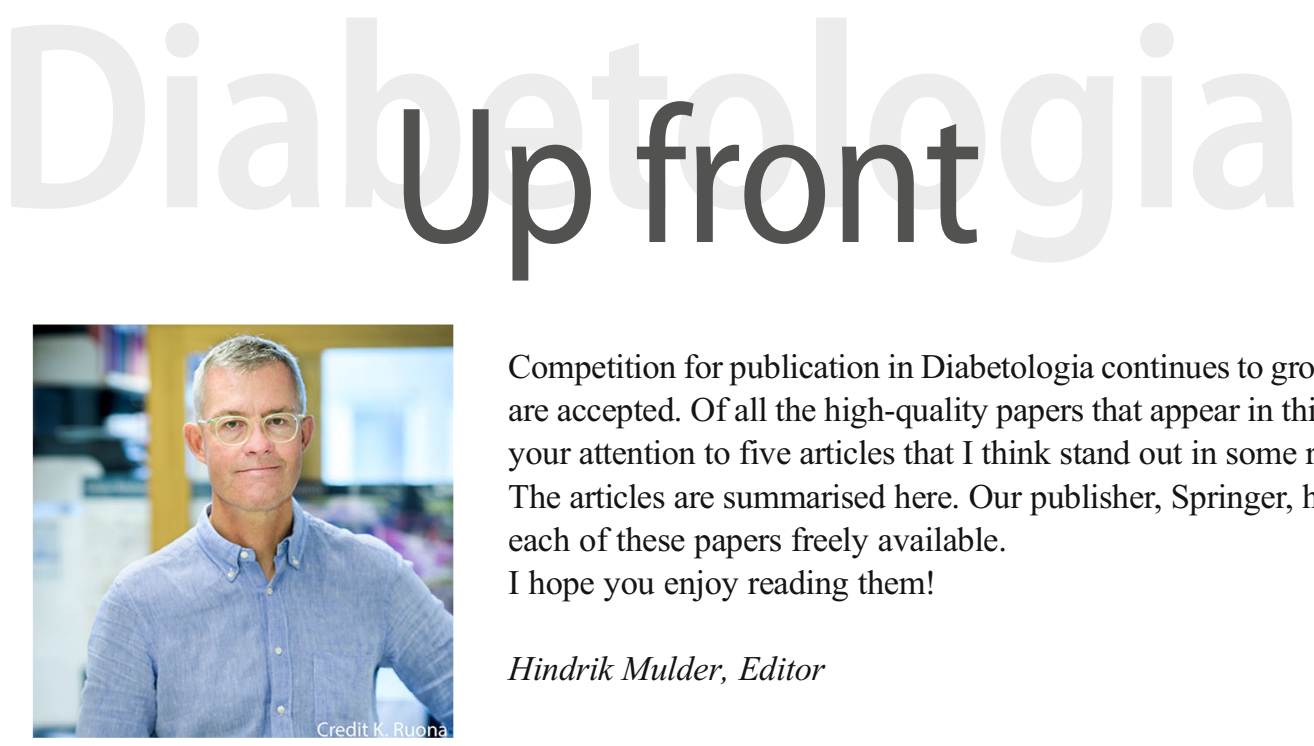

Competition for publication in Diabetologia continues to grow, and less than $20 \%$ of papers are accepted. Of all the high-quality papers that appear in this month's issue I want to draw your attention to five articles that I think stand out in some regard and are very interesting. The articles are summarised here. Our publisher, Springer, has kindly made the full text of each of these papers freely available.

I hope you enjoy reading them!

Hindrik Mulder, Editor

Social networks and type 2 diabetes: a narrative review

Miranda T. Schram, Willem J. J. Assendelft, Theo G. van Tilburg, Nicole H. T. M. Dukers-Muijrers

Social networks are important determinants for well-being and general health in type 2 diabetes. In this issue, Schram et al (https://doi.org/10.1007/s00125-021-05496-2) review the stateof-the-art evidence on the role of social networks on type 2 diabetes risk, diabetes management and the risk for diabetes complications. Despite the heterogeneity of social networks and study designs, the authors reveal that valuable conclusions can be drawn from previous research. For example, living alone and lack of social support have been identified as risk factors for type 2 diabetes. In addition, a smaller network size and less social support increase the risk for diabetes complications, such as chronic kidney disease and CHD. Moreover, the authors highlight that some evidence was found that social support may have a beneficial effect on diabetes self-management. The authors conclude that these findings support the idea that engaging the social network in prevention and treatment strategies for type 2 diabetes may be helpful to improve their effectiveness. The figures from this review are available as a downloadable slideset.

HDL and type 2 diabetes: the chicken or the egg?

Eva Xepapadaki, Ioanna Nikdima, Eleftheria C. Sagiadinou, Evangelia Zvintzou, Kyriakos E. Kypreos

Clinical and epidemiological evidence suggests an optimal range of plasma HDL-cholesterol concentrations, while very low and very high HDL-cholesterol levels are equally associated with high risk of mortality. In addition to plasma HDL-cholesterol levels, the recent observation that the HDL proteome dictates its lipidome, and subsequently HDL particle functionality, indicates that alterations in the HDL metabolic pathway may substantially influence its properties. In this issue, Xepapadaki et al (https://doi.org/10.1007/s00125-021-05509-0) review the accumulating evidence that suggests a bidirectional correlation between HDL dysfunction and type 2 diabetes, leading to a perpetual cycle. Although not all HDL disturbances are causatively associated with the development and progression of type 2 diabetes, many contribute to reduced secretory performance of beta cells in pancreatic islets and skeletal muscle insulin sensitivity. The interrelation between HDL lipidome, proteome and particle functionality remains a missing part of the puzzle that needs to be solved. The authors conclude that understanding HDL functionality and the factors affecting it in individuals with diabetes will be a crucial step towards better glucose homeostasis. The figure from this review is available as a downloadable slide.

Antihypertensive medication needs and blood pressure control with weight loss in the Diabetes Remission Clinical Trial (DiRECT)

Wilma S. Leslie, Eman Ali, Leanne Harris, C. Martina Messow, Naomi T. Brosnahan, George Thom, E. Louise McCombie, Alison C. Barnes, Naveed Sattar, Roy Taylor, Michael E. J. Lean

A diagnosis of type 2 diabetes carries a life-expectancy reduction ranking alongside major cancers, with several weight-related 
cardiometabolic factors (the metabolic syndrome) contributing to this. For example, over half of individuals with type 2 diabetes are also hypertensive. In this issue, Leslie et al (https://doi.org/10. 1007/s00125-021-05471-x) present a secondary analysis of the Diabetes Remission Clinical Trial (DiRECT), in which they aimed to evaluate the safety and efficacy of the planned therapeutic withdrawal of all antihypertensive and diuretic medications, upon commencement of a formula low-energy diet replacement, targeting remission of type 2 diabetes. The authors report that the DiRECT protocol led to remission of diabetes (i.e. $\mathrm{HbA}_{1 \mathrm{c}}$ values that were not in the diabetic range, without glucose-lowering medications) after 2 years for about one-third of all participants who commenced a dietary weight management programme. Over $80 \%$ of those who lost over $15 \mathrm{~kg}$ of weight achieved remission. To avoid postural hypotension, the trial protocol included a therapeutic trial of withdrawing antihypertensive medications upon starting 12-20 weeks of a $3470 \mathrm{~kJ} /$ day (830 kcal/day) formula diet; no rebound hypertension was observed. Overall, blood pressures fell, with $28 \%$ needing to resume antihypertensives before 20 weeks. The weight-loss maintenance protocol maintained normal blood pressure at 24 months, without medication, for $28 \%$ of those who stopped antihypertensives at baseline, and for $44 \%$ of those who sustained remission of diabetes (mean weight loss: $11.4 \mathrm{~kg}$ ). The authors conclude that weight loss is a safe and effective antihypertensive treatment.

Are people with metabolically healthy obesity really healthy? A prospective cohort study of $381,363 \mathrm{UK}$ Biobank participants

Ziyi Zhou, John Macpherson, Stuart R. Gray, Jason M. R. Gill, Paul Welsh, Carlos Celis-Morales, Naveed Sattar, Jill P. Pell, Frederick K. Ho

People who are obese and with a normal metabolic profile are sometimes referred to as having 'metabolically healthy obesity' (MHO). In this issue, Zhou et al (https://doi.org/10.1007/ s00125-021-05484-6) present findings from a prospective cohort study of 381,363 UK Biobank participants. They found that those with $\mathrm{MHO}$ had higher risk of incident diabetes, atherosclerotic cardiovascular disease, heart failure, respiratory disease and all-cause mortality, compared with those who were not obese and had a normal metabolic profile.
The results were similar in an analysis excluding participants with incident outcomes in the first 5 years of follow-up, indicating minimal reverse causation. There were weak or nonsignificant interactions between obesity and metabolic health factors with health outcomes, suggesting $\mathrm{MHO}$ is not a distinctive phenotype. The authors conclude that the use of the label 'metabolically healthy' is misleading and should be avoided. Weight management could be beneficial for all people with obesity, irrespective of metabolic profile.

\section{Branched-chain amino acid metabolism is regulated by ERR $\alpha$ in primary human myotubes and is further impaired by glucose loading in type 2 diabetes}

Rasmus J. O. Sjögren, David Rizo-Roca, Alexander V. Chibalin, Elin Chorell, Regula Furrer, Shintaro Katayama, Jun Harada, Håkan K. R. Karlsson, Christoph Handschin, Thomas Moritz, Anna Krook, Erik Näslund, Juleen R. Zierath

Branched-chain amino acid (BCAA) metabolism is impaired in type 2 diabetes, as reflected by elevated circulating levels of leucine, isoleucine and valine. Since skeletal muscle is the largest contributor to systemic BCAA oxidation, perturbations in myocyte BCAA metabolism may impact whole-body metabolic homeostasis. In this issue, Sjögren, Rizo-Roca et al (https://doi.org/10.1007/ s00125-021-05481-9) demonstrate that skeletal muscle BCAA catabolism in response to glucose loading is attenuated in type 2 diabetes, revealing that the metabolic inflexibility that characterises type 2 diabetes encompasses BCAA catabolism. Moreover, the authors identify oestrogen-related receptor $\alpha(\mathrm{ERR} \alpha)$ as an essential transcriptional partner of peroxisome proliferator-activated receptor $\gamma$ coactivator- $1 \alpha$ (PGC- $1 \alpha)$ in the regulation of the expression of the BCAA gene set in primary human myotubes. The authors state that these findings highlight the utility of an oral glucose tolerance test to unravel disturbances in both BCAA and glucose metabolism and provide insight into the development of new therapeutic strategies for the treatment of type 2 diabetes.

Publisher's note Springer Nature remains neutral with regard to jurisdictional claims in published maps and institutional affiliations.

All text supplied by the authors. 